\section{Obesity linked to risk of kidney stones}

The risk of symptomatic nephrolithiasis is independently associated with body size and obesity according to a recent study by Taylor et al.

A total of 45,988 men, 93,738 older women (aged 34-59 years at baseline) and 101,877 younger women (aged 27-44 years at baseline) were followed using biennial questionnaires to ascertain measurements of body size, diet, and other nondietary covariates such as family history of kidney stones and use of thiazide diuretics. The relative risk of kidney stone formation was then calculated for categories of body size chosen to examine relative extremes.

Over a total of 2,808,334 person-years of follow-up, 4,827 new symptomatic kidney stones were reported. After adjusting for relevant factors such as age, diet and total fluid intake, increased risk of incident kidney stones was positively associated in both men and women with weight, weight gain, $\mathrm{BMl}$ and waist circumference. For example, the multivariate relative risk in younger women with a $\mathrm{BMI}$ of $\geq 30$ versus those with a BMI of 21.0-22.9 was 2.09 (95\% Cl 1.77-2.48, $P<0.001)$. BMI had the greatest effect on risk in women. This may be due to the fact that women generally have more adipose tissue than men with the same BMI.

The results of this study suggest that adiposity is an important factor in determining risk of kidney stone formation. The causes remain unclear, though they may involve obesitylinked insulin resistance, leading to a urinary environment conducive to the formation of stones.

Original article Taylor EN et al. (2005) Obesity, weight gain, and the risk of kidney stones. JAMA 293: 455-462

\section{Sildenafil therapy for erectile dysfunction in peritoneal dialysis patients}

Erectile dysfunction, which commonly occurs in peritoneal dialysis patients, can be effectively treated with sildenafil, according to a recent study published in the American Journal of Kidney Diseases.

This prospective, randomized, double-blind, placebo-controlled, crossover study included
16 men who had been receiving peritoneal dialysis for 6 months to 5 years and who experienced erectile dysfunction. After an initial 4-week assessment period, patients were randomly assigned to receive $50 \mathrm{mg}$ of sildenafil or placebo for 4 weeks, and were then switched for the remaining 4 weeks to the other treatment. Within each treatment period, after 2 weeks the dose of placebo or sildenafil was increased to $100 \mathrm{mg}$, assuming no side effects were reported.

For the patients who completed the trial ( $n=13$, mean age of 53 years) treatment with sidenafil significantly improved erectile function in comparison with baseline measurements or placebo, as assessed by the International Index of Erectile Function questionnaire. Overall satisfaction and intercourse satisfaction also improved significantly in comparison to baseline, but no significant changes in sexual desire were reported in either treatment group. In response to the Global Efficacy Questionnaire, $75 \%$ of patients reported improvement in erections with sildenafil, compared with only $28 \%$ of patients treated with placebo. Sildenafil therapy was well tolerated: no patients discontinued treatment during study and only one adverse event (a headache) was reported.

Phosphodiesterase type 5 inhibitors such as sildenafil have revolutionized the management of erectile dysfunction, but, according to the authors, this is the first randomized, placebo-controlled, crossover study of this drug to be reported in a renal failure population, with results suggesting that sildenafil is an effective and well-tolerated therapy in this patient group.

Original article Mahon A et al. (2005) The efficacy of sildenafil for the treatment of erectile dysfunction in male peritoneal dialysis patients. Am J Kidney Dis 45: 381-387

\section{CD59 could provide a diagnostic clue to prostate cancer prognosis}

Tumor cells use a variety of mechanisms to evade detection and destruction by the immune system. CD59-a complement regulatory protein that inhibits complement-mediated lysis of target cells-is expressed on the surface of a variety of tumor cell types and might confer complement resistance to these cells. In their 